\title{
Quantum jumps and entropy production
}

\author{
Heinz-Peter Breuel* \\ Fachbereich Physik, Carl von Ossietzky Universität, D-26111 Oldenburg, Germany and \\ Physikalisches Institut, Universität Freiburg, D-79104 Freiburg, Germany
}

(Dated: October 23, 2018)

\begin{abstract}
The irreversible motion of an open quantum system can be represented through an ensemble of state vectors following a stochastic dynamics with piecewise deterministic paths. It is shown that this representation leads to a natural definition of the rate of quantum entropy production. The entropy production rate is expressed in terms of the von Neumann entropy and of the numbers of quantum jumps corresponding to the various decay channels of the open system. The proof of the positivity and of the convexity of the entropy production rate is given. Monte Carlo simulations of the stochastic dynamics of a driven qubit and of a $\Lambda$-configuration involving a dark state are performed in order to illustrate the general theory.
\end{abstract}

PACS numbers: 03.65.Yz, 02.70.Ss, 42.50.Lc

Keywords: open quantum systems, quantum entropy, quantum jumps, stochastic wave function method

\section{INTRODUCTION}

Dynamical processes in dissipative open systems involve, in general, irreversible transformations of nonequilibrium states 1, 2]. An important physical quantity of the theory of non-equilibrium processes is the entropy production, that is the rate at which entropy is produced as a result of irreversible processes [3]. In the classical theory the entropy production provides an important Lyapunov functional of non-equilibrium stationary states and leads to the principle of minimal entropy production. It further enables the determination of non-equilibrium transport properties and Onsager coefficients (for a recent discussion of the classical theory see, e. g. [4, 5]).

In the quantum theory of open systems [6] the entropy production is usually related to the negative derivative of the relative entropy with respect to an invariant state. On the ground of the weak coupling master equation for the open system's density matrix the corresponding expressions can again be shown to have useful convexity properties and to satisfy a minimal entropy production principle [7], as well as a kind of quantum Onsager relations 8]. The concept of relative entropy is also useful in quantum information theory 9], e. g. in the quantification of entanglement 10,11] and of the success of quantum teleportation [12]. A general account of the theory of quantum entropy may be found in [13].

During the past decade there has been considerable interest in the application of stochastic wave function methods to the analysis and simulation of dissipative open quantum systems. By contrast to the conventional description in terms of the reduced density matrix, in these methods one considers an ensemble of pure states whose time-evolution is governed by a stochastic process in the underlying Hilbert space $\mathcal{H}$ of the open system. In a particularly interesting method of

*Electronic address: breuer@theorie.physik.uni-oldenburg.de this kind, developed by several authors 14, 15, 16], the wave function represents a piecewise deterministic process in Hilbert space. This means that the stochastic evolution consists of smooth, deterministic parts which are interrupted by instantaneous quantum jumps. Recently, it has been shown that the stochastic wave function method can be generalized to enable the treatment of non-Markovian quantum processes 17] and of Bosonic [18] and of Fermionic [19] many-body systems by means of an appropriate stochastic representation in the doubled Hilbert space $\mathcal{H} \oplus \mathcal{H}$.

The aim of the present paper is to connect the stochastic state vector dynamics to the production of quantum entropy. To this end, we apply the entropy balance equation of non-equilibrium thermodynamics to the von Neumann entropy of the open system. A quantum jump of the stochastic dynamics is linked to the exchange of an entropy quantum between system and environment. This enables one to express the entropy flux in terms of the random processes which count the numbers of quantum jumps belonging to the various decay modes of the open system. In this way, a stochastic representation of the production rate of quantum entropy is derived from the piecewise deterministic state vector evolution. It will be demonstrated that the obtained expression for the entropy production provides a non-negative and convex functional on the state space of the open system. Furthermore, the stochastic representation is shown to lead to a Monte Carlo simulation method for the determination of the quantum entropy production in open systems.

The paper is organized as follows. In Sec. II we briefly recall the dynamical description of open quantum systems in terms of quantum master equations and of stochastic differential equations. Sec. III contains the construction of the stochastic representation of the entropy production rate as well as the proof of its positivity and convexity. Finally, a discussion of the results and examples of Monte Carlo simulations of the entropy production are given in Sec. IV. 


\section{QUANTUM MASTER EQUATIONS AND PIECEWISE DETERMINISTIC PROCESSES}

We consider an open quantum system with reduced density matrix $\rho=\rho(t)$. The open system is coupled to a heat bath, that is to an external reservoir in a thermal equilibrium state at temperature $T$. The free evolution of the system is described by some Hamiltonian $H_{0}$. Furthermore, the system may be subjected to an external time-dependent perturbation, e. g. an external radiation field represented by a Hamiltonian $H_{p}(t)$. Invoking the Markov approximation, the motion of the reduced system is assumed to be representable in terms of a quantum master equation with a generator $\mathcal{L}_{t}$ in Lindblad form [20, 21, 22] (choosing units such that $\hbar=1$ ),

$$
\frac{d}{d t} \rho=\mathcal{L}_{t}(\rho)=-i\left[H_{0}+H_{p}(t), \rho\right]+\mathcal{D}(\rho) .
$$

Since we allow the perturbation to be explicitly timedependent the dynamics does not, in general, provide a dynamical semigroup. However, since $\mathcal{L}_{t}$ is in Lindblad form for each fixed $t$, the time-evolution defined by Eq. (11) yields a two-parameter family of quantum dynamical maps 23].

The commutator in Eq. (11) represents the unitary part of the motion of the reduced system, while $\mathcal{D}(\rho)$ is a super-operator known as the dissipator of the master equation,

$$
\begin{aligned}
\mathcal{D}(\rho)= & \sum_{i} \gamma_{i}^{-}\left(A_{i}^{-} \rho A_{i}^{+}-\frac{1}{2}\left\{A_{i}^{+} A_{i}^{-}, \rho\right\}\right) \\
& +\sum_{i} \gamma_{i}^{+}\left(A_{i}^{+} \rho A_{i}^{-}-\frac{1}{2}\left\{A_{i}^{-} A_{i}^{+}, \rho\right\}\right) .
\end{aligned}
$$

The Lindblad operators $A_{i}^{ \pm}$provide the coupling to the various decay channels of the open system with corresponding damping rates $\gamma_{i}^{ \pm}$. We shall assume in the following that the master equation (11) has been derived from an underlying microscopic theory through the weakcoupling approximation. This implies that the Lindblad operators are obtained as eigen-operators of the unperturbed system Hamiltonian,

$$
\left[H_{0}, A_{i}^{ \pm}\right]= \pm \omega_{i} A_{i}^{ \pm},
$$

and satisfy $A_{i}^{+}=\left(A_{i}^{-}\right)^{\dagger}$. The relation (3) also implies that

$$
\left[H_{0}, A_{i}^{+} A_{i}^{-}\right]=\left[H_{0}, A_{i}^{-} A_{i}^{+}\right]=0 .
$$

The damping rates $\gamma_{i}^{ \pm}$of the decay modes are determined through certain reservoir correlation functions satisfying the Kubo-Martin-Schwinger condition which leads to the relation

$$
\gamma_{i}^{+}=\exp \left(-\omega_{i} / T\right) \gamma_{i}^{-} .
$$

As mentioned in the introduction the dynamics provided by the master equation (1) can be represented in terms of a piecewise deterministic process $\psi(t)(\mathrm{PDP})$ in the Hilbert space of the open system in the sense that its covariance matrix

$$
\rho(t) \equiv \mathrm{E}[|\psi(t)\rangle\langle\psi(t)|]=\int D \psi D \psi^{*} P[\psi, t]|\psi\rangle\langle\psi|
$$

satisfies the master equation (11). Here, E denotes the expectation value of the stochastic process $\psi(t)$ which is defined in terms of the corresponding time-dependent probability density functional $P[\psi, t]$.

An appropriate stochastic differential equation for the state vector dynamics is given by

$$
\begin{aligned}
d \psi(t)= & -i G_{t}(\psi(t)) d t \\
& +\sum_{i}\left(\frac{A_{i}^{-} \psi(t)}{\left\|A_{i}^{-} \psi(t)\right\|}-\psi(t)\right) d N_{i}^{-}(t) \\
& +\sum_{i}\left(\frac{A_{i}^{+} \psi(t)}{\left\|A_{i}^{+} \psi(t)\right\|}-\psi(t)\right) d N_{i}^{+}(t) .
\end{aligned}
$$

The first term on the right-hand side provides the deterministic evolution periods of the process $\psi(t)$. It corresponds to the deterministic non-linear Schrödinger-type equation $\dot{\psi}=-i G_{t}(\psi)$, where

$$
G_{t}(\psi)=\hat{H} \psi+\frac{i}{2} \sum_{i}\left(\gamma_{i}^{-}\left\|A_{i}^{-} \psi\right\|^{2}+\gamma_{i}^{+}\left\|A_{i}^{+} \psi\right\|^{2}\right) \psi
$$

is a non-linear operator with a non-Hermitian Hamiltonian $\hat{H}=\hat{H}(t)$ given by

$$
\hat{H}=H_{0}+H_{p}(t)-\frac{i}{2} \sum_{i}\left(\gamma_{i}^{-} A_{i}^{+} A_{i}^{-}+\gamma_{i}^{+} A_{i}^{-} A_{i}^{+}\right) .
$$

The quantities $d N_{i}^{ \pm}(t)$ in Eq. (7) are the increments of integer-valued random processes $N_{i}^{ \pm}(t)$ satisfying

$$
\begin{aligned}
d N_{i}^{k}(t) d N_{j}^{l}(t) & =\delta_{i j} \delta_{k l} d N_{j}^{l}(t), \\
\mathrm{E}\left[d N_{i}^{ \pm}(t)\right] & =\gamma_{i}^{ \pm}\left\|A_{i}^{ \pm} \psi(t)\right\|^{2} d t,
\end{aligned}
$$

where $k, l= \pm$. According to relation (10) these increments take on the values 0 or 1 . Moreover, if $d N_{i}^{k}(t)$ is equal to 1 for a particular pair $(i, k)$, all other increments are equal to zero. Thus, in view of the stochastic differential equation (7) the state vector then undergoes an instantaneous quantum jump of the form

$$
\psi(t) \longrightarrow \frac{A_{i}^{k} \psi(t)}{\left\|A_{i}^{k} \psi(t)\right\|} .
$$

The relation (11) states that the expectation values of the increments $d N_{i}^{k}(t)$ increase linearly with the time increment $d t$ and are directly proportional to the damping rates $\gamma_{i}^{k}$ and to the square of the norm of $A_{i}^{k} \psi(t)$. Since the latter depends on time through the deterministic motion, the processes $N_{i}^{k}(t)$ are, in general, inhomogeneous in time. They count the number of quantum jumps of type $(i, k)$, i.e. the number of the jumps (12) with Lindblad operator $A_{i}^{k}$. 


\section{DEFINITION OF THE ENTROPY PRODUCTION RATE}

\section{A. Entropy flux and numbers of quantum jumps}

An appropriate starting point for the definition of the entropy production rate $\sigma$ in an open quantum system is provided by the entropy balance equation,

$$
\sigma=\frac{d S}{d t}+J
$$

Here, $S$ is the von Neumann entropy of the open system (the Boltzmann constant is set equal to 1 ),

$$
S=-\operatorname{tr}\{\rho \ln \rho\} .
$$

The quantity $J$ represents the entropy flux, that is the entropy which flows per unit of time from the open system to the reservoir, in which case we have $J>0$, or from the reservoir to the open system, such that $J<0$. Thus, according to Eq. (13) the quantity $\sigma$ is the rate at which entropy is produced owing to irreversible processes.

In order to define the entropy production rate $\sigma$ we therefore need an expression for the entropy flux $J$. To this end, we recall that the external reservoir causes the wave function to perform instantaneous quantum jumps given by (12). According to Eq. (3) the operator $A_{i}^{ \pm}$induces a transition of the system state in which the energy changes by the amount $\pm \omega_{i}$. Thus, $A_{i}^{+}$describes upward and $A_{i}^{-}$downward transitions of the system. Any quantum jump with jump operator $A_{i}^{+}\left(A_{i}^{-}\right)$corresponds to the absorption (emission) of a quantum $\omega_{i}$ of energy from (into) the reservoir. Because the reservoir is assumed to be in a thermal equilibrium state at temperature $T$, the entropy exchange $\delta S$ associated with a quantum jump can be defined by

$$
\delta S=\frac{\omega_{i}}{T}
$$

To justify this expression one first observes that within the Markov approximation of the reduced system dynamics the reservoir correlation functions decay on a time scale which is - by assumption - short compared to the relaxation time of the open system. It is therefore reasonable to assume that the energy exchange in a quantum jump with operator $A_{i}^{ \pm}$appears as a change $\mp \omega_{i}$ of the heat energy of the reservoir. We further note that the reversible character of the energy exchange through a quantum jump derives from the fact that for each jump with operator $A_{i}^{-}$(emission) there also exists the reverse jump with operator $A_{i}^{+}$(absorption).

Thus we see that in each quantum jump with operator $A_{i}^{ \pm}$an entropy quantum (15) is absorbed from or emitted into the reservoir. The integer-valued process $N_{i}^{ \pm}(t)$ represents the number of quantum jumps with operator $A_{i}^{ \pm}$within the time interval $[0, t]$. It follows that in the time interval $[0, t]$, on the average, the net amount

$$
\sum_{i} \frac{\omega_{i}}{T} \mathrm{E}\left[N_{i}^{-}(t)-N_{i}^{+}(t)\right]
$$

of entropy flows into the reservoir. Consequently, the mean entropy flux is obtained from the expression

$$
J=\sum_{i} \frac{\omega_{i}}{T} \frac{d}{d t} \mathrm{E}\left[N_{i}^{-}(t)-N_{i}^{+}(t)\right] .
$$

This relation finally leads to the following stochastic expression of the entropy production rate of the open system,

$$
\sigma=\frac{d S}{d t}+\sum_{i} \frac{\omega_{i}}{T} \frac{d}{d t} \mathrm{E}\left[N_{i}^{-}(t)-N_{i}^{+}(t)\right]
$$

By use of this expression the entropy production rate $\sigma(t)$ can easily be determined by a Monte Carlo simulation of the stochastic process (17). To this end, one evaluates the von Neumann entropy by means of Eq. (6) and records the numbers $N_{i}^{ \pm}(t)$ of the various quantum jumps carried out during the time interval $[0, t]$.

\section{B. Positivity of the entropy production}

We note the following important properties of the entropy production rate $\sigma$ defined by expression (18). First, it is immediately clear that $\sigma$ is an extensive quantity: When combining statistically independent systems which are coupled to the same reservoir, the additivity of the von Neumann entropy and the additivity of the numbers of quantum jumps is obvious. Second, Eq. (18) implies that $\sigma$ does not depend on the specific representation of the state space of the reduced system, since neither the von Neumann entropy nor the number of quantum jumps of a given type changes under unitary transformations. In particular, the expression (18) is invariant under a transformation to the interaction picture with respect to the system Hamiltonian $H_{0}$. Third, the entropy production $\sigma$ as defined in this way is always non-negative,

$$
\sigma \geq 0
$$

which corresponds to the second law of thermodynamics.

To prove the inequality (19) we first write the entropy production rate as a functional $\sigma=\sigma[\rho]$ of the reduced density matrix $\rho$. Invoking the master equation (1) one finds the time-derivative of the von Neumann entropy,

$$
\frac{d S}{d t}=-\operatorname{tr}\{\mathcal{D}(\rho) \ln \rho\}
$$

To obtain an appropriate expression for the entropy flux we use Eqs. (17) and (11), as well as the representation (6) of the density matrix to get

$$
J=\sum_{i} \frac{\omega_{i}}{T} \operatorname{tr}\left\{\gamma_{i}^{-} A_{i}^{+} A_{i}^{-} \rho-\gamma_{i}^{+} A_{i}^{-} A_{i}^{+} \rho\right\} .
$$

On the other hand, employing definition (2) of the dissipator and the commutation relations (3) and (4) one 
finds

$$
\begin{aligned}
\operatorname{tr} & \left\{H_{0} \mathcal{D}(\rho)\right\} \\
= & \sum_{i} \gamma_{i}^{-} \operatorname{tr}\left\{A_{i}^{+}\left[H_{0}, A_{i}^{-}\right] \rho+\frac{1}{2}\left[A_{i}^{+} A_{i}^{-}, H_{0}\right] \rho\right\} \\
& +\sum_{i} \gamma_{i}^{+} \operatorname{tr}\left\{A_{i}^{-}\left[H_{0}, A_{i}^{+}\right] \rho+\frac{1}{2}\left[A_{i}^{-} A_{i}^{+}, H_{0}\right] \rho\right\} \\
= & -\sum_{i} \omega_{i} \operatorname{tr}\left\{\gamma_{i}^{-} A_{i}^{+} A_{i}^{-} \rho-\gamma_{i}^{+} A_{i}^{-} A_{i}^{+} \rho\right\} \\
= & -T J .
\end{aligned}
$$

The commutation relations (3) also imply that the Gibbs state $\rho_{\mathrm{th}}=\exp \left(-H_{0} / T\right) / Z$ with partition function $Z$ represents a zero-mode of the dissipator of the master equation, that is

$$
\mathcal{D}\left(\rho_{\text {th }}\right)=0 .
$$

Physically, this means that the Gibbs state is a stationary solution of the master equation without perturbation $\left(H_{p}=0\right)$. We express the Hamiltonian $H_{0}$ in terms of the Gibbs state,

$$
-H_{0} / T=\ln \rho_{\mathrm{th}}+\ln Z,
$$

which, together with Eq. (22), enables us to write the entropy flux as follows,

$$
J=\operatorname{tr}\left\{\mathcal{D}(\rho) \ln \rho_{\text {th }}\right\} .
$$

Combining this with Eq. (20) we are finally led to the following expression for the entropy production rate in the state $\rho$,

$$
\sigma[\rho]=-\operatorname{tr}\left\{\mathcal{D}(\rho)\left(\ln \rho-\ln \rho_{\text {th }}\right)\right\} .
$$

The inequality (19) can now be shown in two alternative ways. One way is to apply a theorem by Lindblad 24] to the dynamical semigroup $\Lambda_{t}=\exp (\mathcal{D} t)$ whose generator is identical to the dissipator $\mathcal{D}(\rho)$ of the master equation, and to relate the functional (26) to the time-derivative of the relative entropy with respect to the Gibbs state $\rho_{\text {th }}$. Another possibility is to use Lieb's theorem [25] to conclude that the map $\rho \mapsto-\operatorname{tr}\{\mathcal{D}(\rho) \ln \rho\}$ is a convex functional. Since $\operatorname{tr}\left\{\mathcal{D}(\rho) \ln \rho_{\text {th }}\right\}$ is linear in $\rho$, it follows that the entropy production rate $\sigma=\sigma[\rho]$ represents a convex functional of the density matrix. With the help of this property it is easy to demonstrate that the entropy production is non-negative [7]. In fact, applying the definition of convexity to the functional $\rho \mapsto-\operatorname{tr}\{\mathcal{D}(\rho) \ln \rho\}$ one is led to the following inequality which holds for all $\lambda \in[0,1]$,

$$
\begin{aligned}
& -\operatorname{tr}\left\{\mathcal{D}\left(\lambda \rho+[1-\lambda] \rho_{\text {th }}\right) \ln \left(\lambda \rho+[1-\lambda] \rho_{\text {th }}\right)\right\} \\
& \quad \leq-\lambda \operatorname{tr}\{\mathcal{D}(\rho) \ln (\rho)\}-[1-\lambda] \operatorname{tr}\left\{\mathcal{D}\left(\rho_{\text {th }}\right) \ln \left(\rho_{\text {th }}\right)\right\} .
\end{aligned}
$$

Employing the linearity of $\mathcal{D}(\rho)$ and Eq. (23) we get

$$
-\lambda \operatorname{tr}\left\{\mathcal{D}(\rho) \ln \left(\lambda \rho+[1-\lambda] \rho_{\text {th }}\right)\right\} \leq-\lambda \operatorname{tr}\{\mathcal{D}(\rho) \ln (\rho)\} .
$$

We divide by $\lambda$ and perform the limit $\lambda \rightarrow 0$ to arrive at

$$
-\operatorname{tr}\left\{\mathcal{D}(\rho) \ln \left(\rho_{\text {th }}\right)\right\} \leq-\operatorname{tr}\{\mathcal{D}(\rho) \ln (\rho)\},
$$

which, by virtue of (26), is equivalent to inequality (19).

\section{DISCUSSION AND EXAMPLES}

\section{A. General properties of the entropy production}

It has been shown in the preceding section that the piecewise deterministic process (7) for the stochastic state vector of an open quantum system yields a natural definition of the entropy production as a measure of the degree of the irreversibility of the dynamics. In this definition the entropy flux has been expressed in terms of the random numbers of the various types of quantum jumps corresponding to the decay channels of the open system.

The given proof of the positivity of the entropy production rate demonstrates not only that the entropy production $\sigma$ is non-negative (see Eq. (19)) but also that it is a convex functional and that $\sigma\left(\rho_{\mathrm{th}}\right)=0$ is an absolute minimum of the entropy production (see Eq. (26)). Hence, in the absence of external fields $\left(H_{p}=0\right)$ the entropy production vanishes when the equilibrium state is reached, in accordance with the second law of thermodynamics.

We remark that for vanishing external fields $\sigma$ coincides with the negative time derivative of the relative entropy with respect to the Gibbs state $\rho_{\text {th }}$. This is no longer true when external fields are present. Contrary to the definition of entropy production in terms of the relative entropy, the above definition does not assume the existence of a stationary density matrix.

In the case of a non-vanishing external field $\left(H_{p} \neq 0\right)$ one expects, in general, that the systems stays away from equilibrium and that, therefore, entropy is continuously produced. An important physical situation arises (see the examples below) if there exists a stationary solution $\rho^{\mathrm{s}}$ of the master equation in the interaction picture. Since the expression for the entropy production is not affected by the transformation to the interaction picture one finds that the entropy production in the stationary state is given by the constant value

$$
\sigma^{\mathrm{s}}=\sum_{i} \frac{\omega_{i}}{T} \frac{d}{d t} \mathrm{E}\left[N_{i}^{-}(t)-N_{i}^{+}(t)\right],
$$

where $\mathrm{E}$ denotes the expectation value in the stationary state. In such a stationary non-equilibrium state entropy is thus produced at a constant rate $\sigma^{\mathrm{s}}$. What happens physically is that the energy supplied to the system by the external field is ultimately transferred as heat energy to the reservoir leading to a constant entropy production rate.

It is well-known that the stochastic representation of the master equation (11) through a stochastic process of the form (7) is not unique: In general, one can choose, in an infinite number of different ways, the Lindblad jump operators and the Hamiltonian operator without changing the form of the Lindblad generator $\mathcal{L}_{t}$ of the master equation (1). However, different such choices do change, in general, the stochastic dynamics (17). This fact 
is well understood in the framework of continuous measurement theory according to which different choices for the jump operators correspond to different measurement schemes used to monitor the open system 26, 27]. Our construction of expression (18) for the entropy production rate presupposes, however, that the jump operators $A_{i}^{ \pm}$are eigen-operators of the system Hamiltonian, satisfying the commutation relation (3). It is this property which enables one to establish the connection between the stochastic expression (18) and the functional (26). Physically, it is the eigen-operator property which allows one to associate a certain quantum jump of type $(i, k)$ with the exchange of a quantum $\omega_{i}$ of energy with the thermal reservoir.

In the case of a non-degenerate frequency spectrum $\left\{\omega_{i}\right\}$ the requirement provided by the commutation relations (3) uniquely fixes the jump operators and, thus, the stochastic state vector dynamics. If a certain frequency $\omega_{i}$ is degenerate one has the freedom to replace the corresponding jump operators $A_{i \alpha}^{ \pm}$(the index $\alpha$ labels different Lindblad operators belonging to the same frequency $\omega_{i}$ ) by new operators

$$
\tilde{A}_{i \alpha}^{-}=\sum_{\beta} u_{\alpha \beta} A_{i \beta}^{-}, \quad \tilde{A}_{i \alpha}^{+}=\left(\tilde{A}_{i \alpha}^{-}\right)^{\dagger}
$$

where $u_{\alpha \beta}$ is a unitary matrix. However, in view of Eq. (21) it is immediately clear that expression (18) remains invariant under this replacement, showing the uniqueness of the stochastic expression of the entropy production rate.

\section{B. Entropy production rate of a driven qubit}

We conclude the paper by two examples which illustrate several of the features discussed above. The first example is two-state system [28], a qubit consisting of an excited state $|e\rangle$ and a ground state $|g\rangle$ with corresponding energies $E_{e}$ and $E_{g}$. The qubit is coupled to a thermal reservoir and to a single-mode driving field on resonance with the transition frequency $\omega=E_{e}-E_{g}$. The following discussion will be carried out in the interaction picture. The external field is treated in the rotating wave approximation such that the corresponding interaction Hamiltoninan $H_{p}$ becomes time-independent in the interaction picture.

The stochastic dynamics given by Eq. (7) is defined here through two jump operators, given by the raising and lowering operators

$$
A^{+}=|e\rangle\left\langle g\left|, \quad A^{-}=\right| g\right\rangle\langle e|,
$$

with stochastic processes $N^{ \pm}(t)$ for the numbers of the corresponding jumps. The non-Hermitian Hamiltonian (9]) therefore takes the form

$$
\hat{H}=-\frac{\Omega}{2}\left(A^{+}+A^{-}\right)-\frac{i}{2}\left(\gamma^{-} A^{+} A^{-}+\gamma^{+} A^{-} A^{+}\right)
$$

where the Rabi frequency $\Omega$ is assumed to be real. Equation (27) then yields the following entropy production rate in the stationary state,

$$
\sigma^{\mathrm{s}}=-\frac{\omega}{T} \frac{\Omega^{2}}{\gamma} S_{3}=\frac{\omega}{T} \frac{\gamma^{-}-\gamma^{+}}{2+(\gamma / \Omega)^{2}}
$$

where $\gamma=\gamma^{-}+\gamma^{+}$. Obviously, $\sigma^{\mathrm{s}}$ is proportional to the inversion $S_{3}=\rho_{e e}^{\mathrm{s}}-\rho_{g g}^{\mathrm{s}}$ in the stationary state.

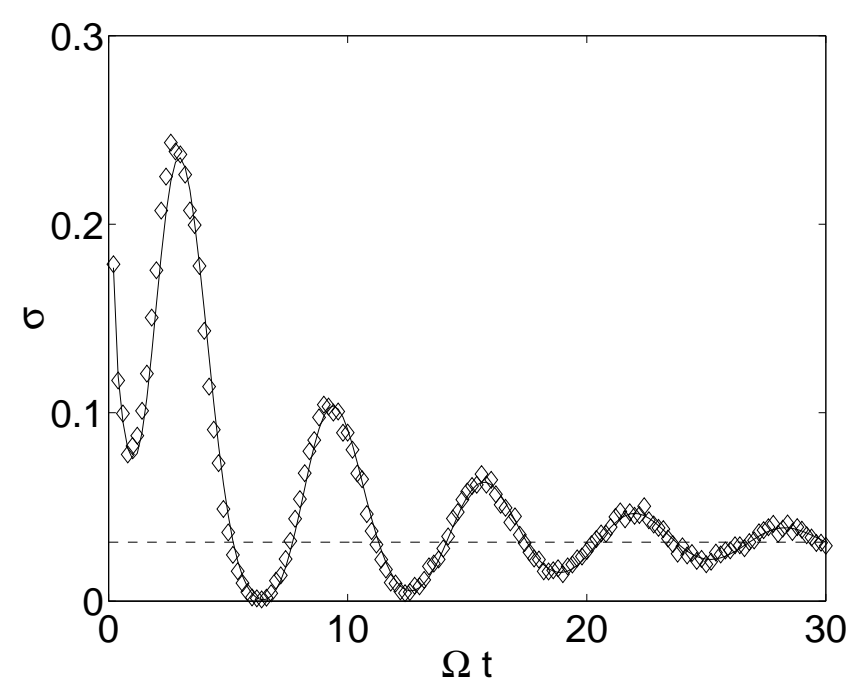

FIG. 1: The entropy production rate $\sigma(t)$ of a driven qubit. Diamonds: Results of a Monte Carlo simulation of the stochastic differential equation (7) using a sample of $10^{5}$ realizations with parameters $\gamma^{-} / \Omega=0.1$ and $\omega / T=1.0$. Continuous line: The entropy production rate obtained from the solution of the master equation (1). Dashed line: The stationary rate $\sigma^{\mathrm{s}}$ according to Eq. (31).

Figure 1 shows the result of a Monte Carlo simulation of the PDP defined by Eq. (7) and compares it with the solution of the density matrix equation (1). In the stochastic simulation the entropy production rate has been determined with the help of Eq. (18), where both the von Neumann entropy $S$ and the expectation values of the numbers $N^{ \pm}(t)$ of quantum jumps have been estimated from a sample of $10^{5}$ realizations of the PDP. The initial condition has been chosen to be the ground state $|g\rangle$ of the qubit. In the absence of the driving field the entropy production decreases to zero during the approach to the thermal equilibrium state. However, as can be seen from the figure, in the presence of an external driving the entropy production oscillates and converges to the stationary value given by Eq. (31).

\section{C. $\Lambda$-configuration with a dark state}

As our second example we investigate a two-level atom with transition frequency $\omega$. Both levels are threefold degenerate forming manifolds with total angular momentum $J_{e}=1$ (excited level) and $J_{g}=1$ (ground level). 
We introduce the energy eigenstates $\left|g, m_{g}\right\rangle$ and $\left|e, m_{e}\right\rangle$ which are simultaneously eigenstates of the $z$-component of the atomic angular momentum operator with eigenvalues $m_{g}$ and $m_{e}$, respectively. In addition to the coupling to a thermal reservoir the atom is subjected to a resonant driving field which is linearly polarized in the $y$-direction. If we take some initial state in the manifold spanned by the states $\left|g, m_{g}= \pm 1\right\rangle$ we then find that the dynamics is confined to the subspace spanned by the basis states

$$
\left|e, m_{e}=0\right\rangle, \quad\left|g, m_{g}=+1\right\rangle, \quad\left|g, m_{g}=-1\right\rangle,
$$

forming a level scheme known as $\Lambda$-configuration [29].

The dynamics of the system will again be studied in the interaction picture. It can be described by the four jump operators,

$$
A_{1}^{-}=\frac{1}{\sqrt{2}}|g,-1\rangle\left\langle e, 0\left|, \quad A_{2}^{-}=\frac{1}{\sqrt{2}}\right| g,+1\right\rangle\langle e, 0|,
$$

and $A_{1}^{+}=\left(A_{1}^{-}\right)^{\dagger}, A_{2}^{+}=\left(A_{2}^{-}\right)^{\dagger}$. The corresponding matrix representation of the non-Hermitian Hamiltonian (9) in the basis (32) reads

$$
\hat{H}=-\frac{i}{2}\left(\begin{array}{ccc}
\gamma^{-} & \Omega & -\Omega \\
-\Omega & \frac{\gamma^{+}}{2} & 0 \\
\Omega & 0 & \frac{\gamma^{+}}{2}
\end{array}\right) .
$$

A numerical simulation of the dynamics is shown in Fig. 2 In the Monte Carlo simulation of the corresponding stochastic differential equation (7) the quantity $\sigma(t)$ has been determined with the help of Eq. (18) by recording the stochastic numbers $N_{1,2}^{ \pm}(t)$ of upward and downward transitions in a sample of realizations of the process.

The stationary entropy production rate may be found from Eq. (27) with the result

$$
\sigma^{\mathrm{s}}=\frac{\omega}{T} \gamma^{+} \rho_{23}^{s}=\frac{\omega}{T} \gamma^{+} \frac{\gamma^{-}-\gamma^{+}}{2 \gamma^{-}+4 \gamma^{+}+\frac{\gamma^{+}}{\Omega^{2}}\left(\gamma^{-}+\frac{\gamma^{+}}{2}\right)^{2}} .
$$

This equation shows that $\sigma^{\mathrm{s}}$ is proportional to the matrix element $\rho_{23}^{s}=\left\langle g,+1\left|\rho^{\mathrm{s}}\right| g,-1\right\rangle$ of the stationary density matrix $\rho^{\mathrm{s}}$. We observe that, similarly to the case of the driven qubit (see Eq. (31)), $\sigma^{\mathrm{s}}$ approaches a finite value as $\Omega \longrightarrow \infty$. For zero Rabi frequency, $\Omega=0$, the stationary entropy production rate vanishes, in accordance with our general discussion. Moreover, the entropy production vanishes for infinite temperatures for both the driven qubit and the $\Lambda$-configuration.

By contrast to the driven qubit, however, in the case of the $\Lambda$-configuration the limit $T \longrightarrow 0$, corresponding to a reservoir at zero temperature, leads to a vanishing entropy production rate. In fact, in this limit we have $\gamma^{-} \longrightarrow \gamma_{0}, \rho_{23}^{s} \longrightarrow 1 / 2$ and, therefore,

$$
\lim _{T \rightarrow 0} \sigma^{\mathrm{s}}=\lim _{T \rightarrow 0} \frac{\omega}{T} e^{-\omega / T} \gamma_{0} \frac{1}{2}=0 .
$$

This behaviour can easily be understood if we note that the matrix element $\rho_{23}$ of the density matrix describes

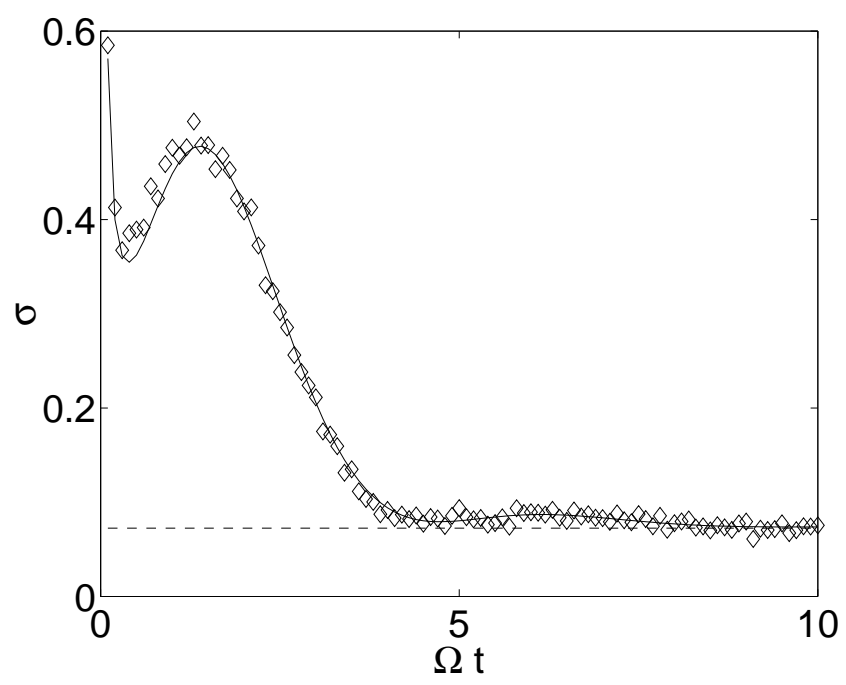

FIG. 2: The entropy production rate $\sigma(t)$ of a driven $\Lambda$ configuration involving a dark state. Diamonds: Monte Carlo simulation of the corresponding stochastic differential equation (7) with $10^{5}$ realizations and parameters $\gamma^{-} / \Omega=1$ and $\omega / T=1.25$. Continuous line: The entropy production rate obtained from the solution of the master equation. Dashed line: The stationary entropy production rate $\sigma^{\mathrm{s}}$ given in Eq. (35).

the coherence of the state

$$
\left|\psi_{\mathrm{d}}\right\rangle=\frac{1}{\sqrt{2}}(|g,+1\rangle+|g,-1\rangle)
$$

This state is known as dark state since it does not couple to the driving field on account of a quantum interference effect.

According to Eq. (35) the stationary entropy production rate is thus proportional to the coherence of the dark state in the stationary state $\rho^{\mathrm{s}}$. At zero temperature, any initial state is driven into the dark state under the dynamics. The reason of this fact is that $\left|\psi_{\mathrm{d}}\right\rangle$ represents a zero-mode of the non-Hermitian Hamiltonian (34) for $T=0$, that is for $\gamma^{+}=0$. Equation (36) therefore states that the entropy production rate vanishes in the dark state. This follows immediately from the fact that $\left|\psi_{\mathrm{d}}\right\rangle$ belongs to the zero temperature ground state manifold.

Finally, we remark that this system provides an example of a system with a degenerate frequency spectrum, mentioned at the end of Sec. IVA Both jump operators given in (33) belong to the same transition frequency $\omega$. In a continuous measurement interpretation they correspond to a measurement of the angular momentum of the emitted quanta along the $z$-direction [30]. The measurement along any other direction amounts to a transformation of these jump operators which is of the form (28) and which does not change the expression for the entropy production rate. The latter is therefore seen to be independent of the measurement scheme. 
[1] S. R. de Groot and P. Mazur, Non-Equilibrium Thermodynamics, (North-Holland, Amsterdam, 1962).

[2] J. Keizer, Statistical Thermodynamics of Nonequilibrium Processes, (Springer-Verlag, New York, 1987).

[3] G. Nicolis and I. Prigogine, Self-Organization in Nonequilibrium Systems, (Wiley, New York, 1977).

[4] D. Daems and G. Nicolis, Phys. Rev. E 59, 4000 (1999).

[5] Bidhan Chandra Bag, Phys. Rev. E 66, 026122 (2002).

[6] H. P. Breuer and F. Petruccione, The Theory of Open Quantum Systems, (Oxford University Press, Oxford, 2002).

[7] H. Spohn, J. Math. Phys. 19, 1227 (1978).

[8] K. Lendi, Phys. Rev. A 34, 662 (1986).

[9] M. A. Nielsen and I. L. Chuang, Quantum Computation and Quantum Information, (Cambridge University Press, Cambridge, 2000).

[10] L. Henderson and V. Vedral, Phys. Rev. Lett. 84, 2263 (2000).

[11] K. Audenaert, B. De Moor, K. G. H. Vollbrecht, and R. F. Werner, Phys. Rev. A 66, 032310 (2002).

[12] G. Bowen and S. Bose, Phys. Rev. Lett. 87, 267901 (2001).

[13] A. Werl, Rev. Mod. Phys. 50, 221 (1978).

[14] J. Dalibard, Y. Castin, and K. Mølmer, Phys. Rev. Lett. 68, 580 (1992).

[15] R. Dum, P. Zoller, and H. Ritsch, Phys. Rev. A 45, 4879 (1992).

[16] H. Carmichael, An Open Systems Approach to Quantum
Optics, Lecture Notes in Physics m18 (Springer-Verlag, Berlin, 1993).

[17] H. P. Breuer, B. Kappler, and F. Petruccione, Phys. Rev. A 59, 1633 (1999).

[18] I. Carusotto, Y. Castin, and J. Dalibard, Phys. Rev. 63, 023606 (2001).

[19] O. Juillet and Ph. Chomaz, Phys. Rev. Lett. 88, 142503 (2002).

[20] E. B. Davies, Commun. Math. Phys. 39, 91 (1974).

[21] V. Gorini, A. Kossakowski, and E. C. G. Sudarshan, J. Math. Phys. 17, 821 (1976).

[22] G. Lindblad, Commun. Math. Phys. 48, 119 (1976).

[23] R. Alicki and K. Lendi, Quantum Dynamical Semigroups and Applications, Volume 286 of Lecture Notes in Physics, (Springer-Verlag, Berlin, 1987).

[24] G. Lindblad, Commun. Math. Phys. 40, 147 (1975).

[25] E. H. Lieb, J. Math. Phys. 14, 1938 (1973).

[26] H. M. Wiseman and G. J. Milburn, Phys. Rev. A 47, 642 (1993).

[27] H. M. Wiseman and G. J. Milburn, Phys. Rev. A 47, 1652 (1993).

[28] C. W. Gardiner and P. Zoller, Quantum Noise, second edition, (Springer-Verlag, Berlin, 2000).

[29] M. O. Scully and M. S. Zubairy, Quantum Optics, (Cambridge University Press, Cambridge, 1997).

[30] K. Mølmer, Y. Castin, and J. Dalibard, J. Opt. Soc. Am. B 10, 524 (1993). 